\title{
Low moduli elastomers with low viscous dissipation
}

\author{
Bejenariu, Anca Gabriela; Yu, Liyun; Skov, Anne Ladegaard
}

Published in:

Soft Matter

Link to article, DOI:

10.1039/c2sm25134e

Publication date:

2012

Link back to DTU Orbit

Citation (APA):

Bejenariu, A. G., Yu, L., \& Skov, A. L. (2012). Low moduli elastomers with low viscous dissipation. Soft Matter, 8(14), 3917-3923. https://doi.org/10.1039/c2sm25134e

\section{General rights}

Copyright and moral rights for the publications made accessible in the public portal are retained by the authors and/or other copyright owners and it is a condition of accessing publications that users recognise and abide by the legal requirements associated with these rights.

- Users may download and print one copy of any publication from the public portal for the purpose of private study or research.

- You may not further distribute the material or use it for any profit-making activity or commercial gain

- You may freely distribute the URL identifying the publication in the public portal

If you believe that this document breaches copyright please contact us providing details, and we will remove access to the work immediately and investigate your claim 


\title{
Low moduli elastomers with low viscous dissipation
}

\author{
Anca Gabriela Bejenariu, Liyun Yu, Anne Ladegaard Skov*
}

\author{
5 Received (in $X X X, X X X) X$ th $X X X X X X X X X 20 X X$, Accepted $X$ th $X X X X X X X X X 20 X X$ \\ DOI: 10.1039/b000000x
}

A controlled reaction schema for addition curing silicones leads both to significantly lower elastic modulus and lower viscous dissipation than for the chemically identical network prepared by the traditional reaction schema.

\section{${ }_{10}$ Introduction}

An elastomer is a crosslinked polymeric material with the capacity of returning to its original size and shape after being deformed but is not as such regarded as a so-called 'smart material' which is defined as a material which changes one or 15 more physical property upon an external stimulus such as $\mathrm{pH}$, temperature, light, magnetic or electric fields. However, when the elastomer is used as electroactive polymer (EAP), in which the elastomer is sandwiched between two compliant electrodes, it can be regarded as a smart composite material with a shape response 20 to external voltage.

An ideal elastomer for EAP uses is characterized by high extensibility, flexibility and good mechanical fatigue as well as high electrical and mechanical breakdown strengths. The elastomer usually consists of a polymer network and different

${ }_{25}$ fillers to enhance the mechanical breakdown strength as well as the tear strength. A polymer network is a three-dimensional entity of polymer chains connected by covalent bonds which are introduced by the reaction of a polymer with a crosslinker. Most model elastomers are prepared by an end-linking process using a 30 crosslinker with a certain functionality $f$ and a linear polymer with functional groups in both ends, and the resulting networks are so-called unimodal networks where unimodal refers to the number of polymers with different average molecular weights (in the unimodal case one polymer is used). The resulting network 35 contains different structures, namely 1 ) the elastically active network chains, 2) the dangling chains and substructures, and 3) the so-called sol fraction where the latter structure is not chemically attached to the network but rather acts as a solvent $t^{1,2}$. The dangling chains and the sol fractions are contributing to the 40 softness of the network and result in higher extensibility but lower the mechanical breakdown strength significantly. As an alternative to the unimodal networks, two polymers with significantly different average molecular weights can be mixed together with one crosslinker, and the networks are termed 45 bimodal networks ${ }^{3}$. If the polymers have similar molecular weights it can be discussed whether the network is unimodal or bimodal as the term unimodal is not strictly defined with respect to the polydispersity (i.e. how broadly the chain lengths of the polymer are distributed) so in the following we assume that the 50 average molecular weights of the two polymers always are significantly different (at least a factor of 5) and that the polydispersity of each polymer is less than 2 . The preparation of bimodal networks opens up for the introduction of a controlled reaction procedure which allows for the formation of a 55 heterogeneous network. The reaction procedure first allows for the short chains to react with deficient amounts of crosslinker to form hyperbranched structures which are then reacted with the remaining crosslinkers and the long polymers. Illustrations of the homogeneous and heterogeneous bimodal networks can be seen ${ }_{60}$ in Figures 1a and 1b, respectively. A homogeneous bimodal network refers to an almost uniform distribution of the short chains and the long chains in the network, being obtained by simultaneously mixing of the chains with a certain quantity of the crosslinker.

${ }_{65}$ Heterogeneous bimodal networks have been shown to act in a surprisingly different way than the homogeneous bimodal networks ${ }^{3,4}$. The clusters act as 'elastic crosslinkers' and at small deformations the apparent crosslinkers will be the clusters and at large deformations the apparent crosslinkers will be the true 70 crosslinkers. In other words the network will be relatively soft up to the point where the stress becomes sufficiently large to initiate the deformation of the clusters. Therefore the bimodal heterogeneous network will reinforce itself at large strains and hence the mechanical breakdown strength may become very large 75 as long as the breakdown strength of the material surrounding the clusters is larger than the strength needed to initiate the deformation of the clusters.
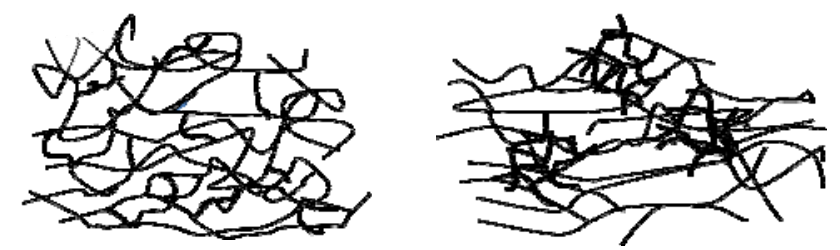

Figure 1a. Schematic Figure 1b. Schematic illustration of illustration of a bimodal a bimodal network with a network with a non- controlled clustered distribution of controlled distribution of the short polymeric chains the short polymeric chains

While several types of EAPs have been investigated ${ }^{5-8}$ most effort has been put into dielectric elastomers (DE), which have been 80 shown to be particularly attractive for large strain and high-power applications ${ }^{9-11}$. Silicone and acrylic based elastomers are the most commonly used material as EAPs.

$\mathrm{VHB}^{\mathrm{TM}}$ acrylic elastomers from $3 \mathrm{M}$ have been widely used in actuators and have shown good performance but they rely on ${ }_{85}$ prestretching which is not favourable for applications that rely on 
constant actuation over a longer period as the prestretch effect tends to decrease over time ${ }^{12}$. Silicone rubber films based on poly(dimethylsiloxane) (PDMS) and a compatible crosslinker have also been used with good results as electrically responsive 5 materials ${ }^{13}$. However, the commercial applicability of silicones with properties fulfilling the DE requests are limited (e.g. CF192186 from Nusil, Elastosil RT625 from Wacker Chemie, and KE441 from ShinEtsu), with few possibilities for optimization to specific uses. Kornbluh made a comparison of different 0 parameters between the two most commonly used polymers ${ }^{14}$. The acrylic based materials are favoured by characteristics like large strains, high forces and high energy density whereas the silicones are favoured by fast responses, broad temperature stability and less viscous dissipation ${ }^{14}$.

15 Actuators are transducers that convert electrical energy into mechanical energy and they consist of dielectric elastomers sandwiched between two compliant electrodes (figure 2). If a voltage is applied between the two electrodes, the charges from the electrodes will attract each other and create an electrostatic

20 field. The mechanical compression that acts normal to the film surface is defined as Maxwell stress and it determines the decrease of the thickness of the film $\left(\mathrm{d}_{1}>\mathrm{d}_{2}\right)$ and the enlargement of the surface area $\left(\mathrm{A}_{1}<\mathrm{A}_{2}\right)$ as illustrated in Figure 2. The transducer can also be operated in the reverse fashion and is then

25 referred to as a generator as a voltage is produced from the motion of the material.

The actuation mechanism may be explained as the electromechanical response of EAPs to the development of a Maxwell stress under an electrical field. Depending on the 30 properties of the material (Poisson's ratio, dielectrical permittivity and elasticity) and the magnitude of the electrical field, the Maxwell stress may induce a significant shape modification of the elastomer.

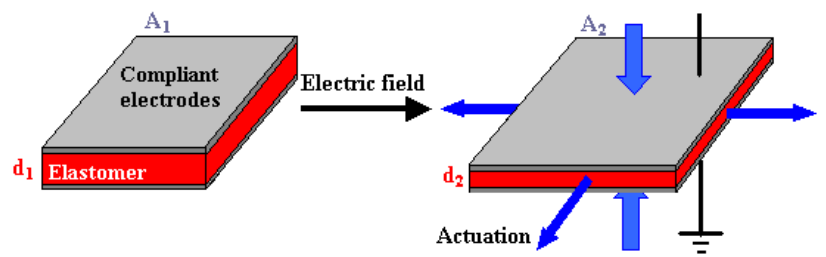

35 Figure 2. Schematic illustration of the operational principle for a conventional dielectric elastomer before $\left(A_{1}, d_{1}\right)$ and after $\left(A_{2}, d_{2}\right)$ introduction of an external voltage pulse ${ }^{15}$

The changes in the dimensions of the elastomer are estimated by assuming that the stress, $s$, is proportional to the strain, $p$, 40 perpendicular to the stress:

$$
s=Y \cdot p \quad(\text { eq. } 1)
$$

where $Y$ is the Young modulus. The electrostatic pressure from the charges on the compliant electrodes $\left(p_{\mathrm{el}}\right)$, the so-called Maxwell stress, is defined as:

$$
p_{e l}=\varepsilon_{0} \cdot \varepsilon_{r} \cdot E^{2}=\varepsilon_{0} \cdot \varepsilon_{r} \cdot\left(\frac{U}{d_{1}}\right)^{2} \text { (eq. 2) }
$$

where $\varepsilon_{0}$ is the vacuum permittivity $\left(8.85 \cdot 10^{-12} \mathrm{~F} / \mathrm{m}\right), \varepsilon_{\mathrm{r}}$ is the relative dielectric constant of the elastomer and $\mathrm{E}$ is the applied electrical field ${ }^{16}, U$ is the voltage and $d_{1}$ is the initial thickness of the material. At equilibrium the electrostatic pressure equals the ${ }_{50}$ internal stress in the material such that the strain (actuation) can be written as:

$$
p=\frac{\varepsilon_{0} \cdot \varepsilon_{r}}{Y}\left(\frac{U}{d_{1}}\right)^{2} \text { (eq. 3) }
$$

When the voltage is switched off, the electrodes are shortcircuited and the material goes back to its initial shape $\left(d_{1}, A_{1}\right)$. ${ }_{55}$ Generally the required voltage for a significant actuation is too high and for more general applicability of EAPs it is a requirement that the voltage is lowered. The applied voltage for a given actuation can be decreased by changing the relative permittivity and the thickness of the film. From eq. 3 it can be ${ }_{60}$ seen that it is more effective to decrease the thickness of the film than to increase the electrical permittivity but the reduction of the thickness causes problems with processing and general handability of the films and hence the thickness is not always favourable as a tuning parameter.

${ }_{65}$ Viscoelasticity plays a significant role in the performance and reliability of the EAPs ${ }^{17}$. For reliability of the EAP, a displacement attenuation caused by viscous dissipation can not be accepted. Several failure modes of the EAP also depend on the viscoelastic behaviour of the elastomer. Plante and Dubowsky ${ }^{18}$ 70 developed a model to understand the nature of EAP failure and thereby be able to give guidelines on how to avoid failure. To predict EAP failure modes, the model needs to include the effects of very large deformations on both the viscoelasticity and the dielectric strength of the polymer network. The developed model 75 predicts EAP actuation (stretch) as a function of prestretch, applied voltage, stretch rate, and work load. Three failure criteria are investigated by Plante and Dubowsky: (1) material strength failure that is principally dominated by film stretch, (2) dielectric strength failure determined by the breakdown voltage limit of the 80 material and (3) pull-in failure that appears when Maxwell stress is higher than the internal stress. At high stretch rates, the lack of dielectric strength is the dominant mode of failure. At low stretch rates, pull-in is the dominating mode of failure. An important practical result of the performed study is that EAPs have failure 85 modes and performance boundaries that depend strongly on stretch rate ${ }^{18}$. Hence the nonlinear rheological properties of the film play an important role in all three failure modes. The failure modes are schematically illustrated in Figure 3.

$$
\text { material strength }
$$

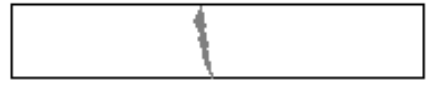

dielectric strength

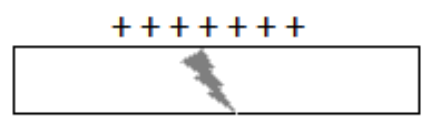

pull-in

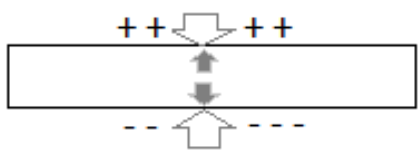

90 Figure 3. Schematic representation of failure modes for an EAP in an actuator system. The failure modes appear at a critical voltage

To ensure large actuation and cyclic displacement of the EAP, the materials must 1) posses high mechanical strength as a result of 95 the chemical or physical crosslinking, 2) be flexible with a small elastic modulus, 3) possess a moderate-to-high dielectric constant (typical $\varepsilon$ values range from 3 to 7$)^{5,19}$ and 4) be characterized by a high electrical breakdown value. The electrical breakdown can be defined as a sudden decrease of resistivity of a material when 
the applied electric field strength rises above a certain threshold value (the dielectric strength of the material). To deform a material in a substantial way, it has to be soft, but in this situation the system is susceptible of electromechanical instability. Hence, 5 an optimum between mechanical and electrical breakdown must be found in order to have an ideal EAP. The high tear strength is, however, not always strictly required for e.g. EAPs prepared by micro fabrication where the EAP is built up by many layers ${ }^{20}$.

In general elastomers should be mechanically characterized in 10 terms of both linear and nonlinear elongation to evaluate the material properties, but measurements are difficult to perform and time-consuming for soft networks ${ }^{21-23}$. The linear viscoelastic (LVE) diagrams can be obtained from commercial shear and strain controlled rheometers and provides information about the 5 elastic modulus and the degree of viscous dissipation at low strains and may be sufficient for many applications ${ }^{24}$.

An optimization of the mechanical properties of the elastomer is possible by varying the mass ratio between the short and long polymeric chains or by tuning the stoichiometry of the reactive 20 components. The elastomer properties are intended to be improved via two preparation processes inducing lowering of the elastic modulus without compromising the other required mechanical properties (fast response, stability, low degree of viscous dissipation, high extensibility) and without changing

25 permittivity as well. Usually for unimodal networks, a low elastic modulus means a network with no mechanical strength as well, thereby causing problems with both the handling of the film and the tear strength of the material.

Ouyang et al made a thorough study of the elastic modulus and 30 response time as function of the stoichiometry where they obtained elastic moduli in the range from 1 to $600 \mathrm{kPa}^{25}$. The change of stoichiometry to the vicinity of the gelation thresholds is, however, not an option for DEAP purposes since the inreacted polymer (or crosslinker close to the upper gelation threshold) will 35 constitute a significant fraction even relatively far from the gelation threshold ${ }^{1,26}$. This may cause a problem in both largescale processing of DEAP as deposition of the electrodes may result due to the inreacted PDMS which will act as an oil and in the usage of the DEAP where the PDMS may migrate away from 40 the system causing both long-term delamination of electrodes and increased elastic modulus of the material ${ }^{27}$.

The heterogeneous bimodal networks constitute a system with many degrees of freedom and hence provide a greater possibility for optimization to specific uses. The degrees of freedom include 45 amongst other the overall stoichiometry, the functionality of the crosslinker and the molecular weight of the long polymer as for the unimodal network but also the ratio between the short and the long chains, the stoichiometry of the pre-mixes, the molecular weight of the short polymer, the amount of solvent, and the ${ }_{50}$ different waiting times.

\section{Materials and methods}

The networks are synthesized from polydimethyl siloxanes (PDMS) of different molecular weight: DMS-V05 with $\mathrm{M}_{\mathrm{n}}=800$ $\mathrm{g} / \mathrm{mol}$, DMS-V22 with $\mathrm{M}_{\mathrm{n}}=9400 \mathrm{~g} / \mathrm{mol}$, DMS-V35 with ${ }_{55} \mathrm{M}_{\mathrm{n}}=49500 \mathrm{~g} / \mathrm{mol}$ and DMS-V41 with $\mathrm{M}_{\mathrm{n}}=62400 \mathrm{~g} / \mathrm{mol}$ and a low molecular weight crosslinker (X-linker): tetrakis(dimethyl siloxy)-silane with $\mathrm{M}_{\mathrm{w}}=328 \mathrm{~g} / \mathrm{mol}$. All the polymers are purchased from Gelest Inc. and the given molecular weights are supplied by the company. The catalyst (platinum 60 cyclovinylmethyl siloxane complex (511)) is provided by Hanse Chemie.
The unimodal and the bimodal networks are prepared by using 2 or 4 pre-premixes, respectively. The pre-premixes A contain PDMS and crosslinker, while the pre-premixes B contain PDMS 65 and catalyst. The purpose of the premix and the pre-premix preparation is to ensure sufficient mixing of crosslinker and polymer, and catalyst and polymer, respectively ${ }^{22,28}$. Finally, the pre-premixes are mixed in different mass ratios corresponding to different stoichiometric imbalances. The stoichiometric 70 imbalance, $r$, is defined as the ratio of the reactive groups of the PDMS $(-\mathrm{CH}=\mathrm{CH} 2)$ to the reactive groups of the crosslinker (-SiH) ${ }^{1}$.

The unimodal networks consist of either PDMS-V35 and PDMSV41 and the 4-functional crosslinker by use of the 'one-step two75 pot' $(1 \mathrm{~s}-2 \mathrm{p})$ procedure. A conceptualization of the procedure is presented in Figure 4. The unimodal networks are prepared by mixing the premixes $\mathrm{A}$ and $\mathrm{B}$ in different mass ratios. The reaction takes place at ambient conditions for at least 5 hours.

Further details about the unimodal network preparation can be 80 found in Bejenariu et al. ${ }^{22}$.

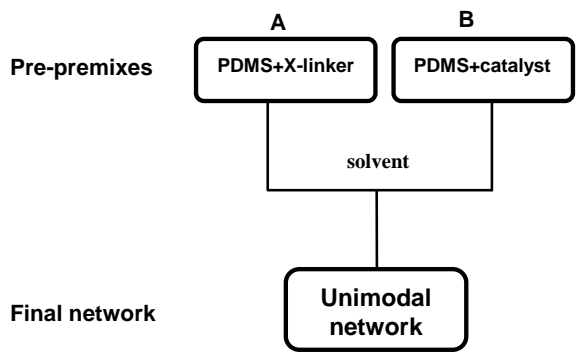

Figure 4. Conceptualization of the 'one-step two-pot' (1s-2p) synthesis procedure of the unimodal networks

The bimodal networks are prepared using either a 'one-step four${ }_{85}$ pot' $(1 \mathrm{~s}-4 \mathrm{p})$ or 'two-step four-pot' $(2 \mathrm{~s}-4 \mathrm{p})$ mixing procedures. The conceptualization of the two methods is shown in Figure 3 and 4 , respectively.

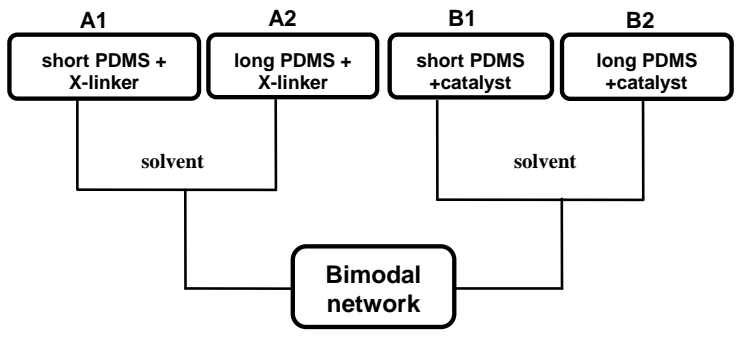

Figure 5. Conceptualization of the 'one-step four-pot' (1s-4p) 90 synthesis procedure of the bimodal networks

Four pre-premixes are prepared: 2 pre-premixes with short PDMS chains (PDMS-V05 or PDMS-V22) (A1 and B1) and 2 prepremixes with long PDMS chains (PDMS-V35 or PDMS-V41) (A2 and B2). A small amount of solvent is added to the premixes 95 to avoid the presence of air bubbles in the final network and to facilitate mixing. Heptane is used as solvent in small quantities (max. 50\% solvent) and degassing is employed to remove the dissolved gasses. 


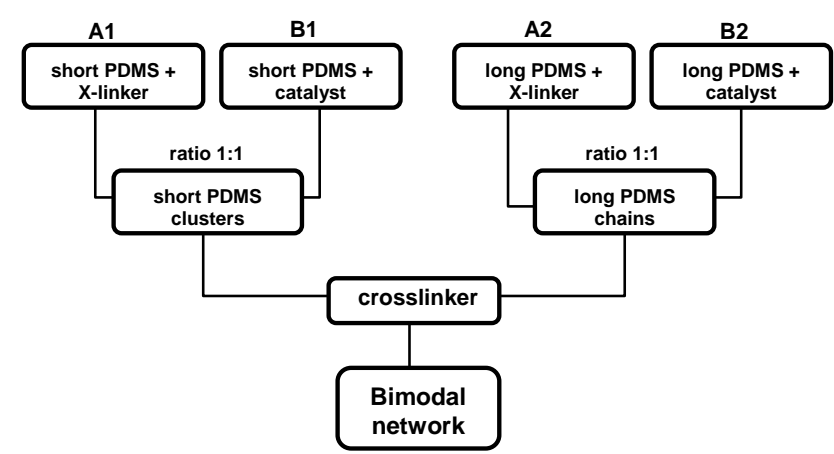

Figure 6. Conceptualization of the 'two-step four-pot' $(2 s-4 p)$ synthesis procedure of the bimodal networks

For the (1s-4p) procedure the pre-premixes are mixed two by two 5 (A1 with $\mathrm{A} 2$ and $\mathrm{B} 1$ with $\mathrm{B} 2$ ) before mixing the entire system together (Figure 5). For the $(2 s-4 p)$ procedure the pre-premixes are mixed two by two (A1 with B1 and A2 with B2) in ratios which corresponds to $\mathrm{r}=0.30$ for both short and long chain based premixes (Figure 6). The stoichiometric imbalance of both short 10 and long chain base pre-network is chosen below the gel point of the system. The critical stoichiometry $\left(r_{c}\right)$ (the gel point) is determined from the Flory-Stockmayer expression: $r_{c}=1 /(f-1)^{29,} 30$ The four functional crosslinker gives a critical stoichiometry of $r_{c}=0.33$.

${ }_{15}$ In the second step of the $2 s-4 p$ procedure (Figure 6) the two premixes are mixed in certain amounts according to the final mass ratio between short and long chains (short:long $=97: 3$, $90: 10,80: 20$ or $20: 80,10: 90,3: 97$ ) and the required quantity of crosslinker is added in order to have a final bimodal network with 20 a certain overall $\mathrm{r}$.

For the networks with fillers, treated silica particles $\left(\mathrm{SiO}_{2}\right.$, hexamethyldisilazane treated, Gelest Inc.) are used to increase the tear strength. The given amount of particles is mixed with solvent in a ratio of $1 \mathrm{~g}$ particles to $1.5 \mathrm{~mL}$ heptane. The mixtures are 25 mechanically stirred and ultra-sonicated for 30 minutes. Finally, the filler solution is added to the long chain mixture and mixed for 10 minutes.

Thin films are prepared using an in-house designed coating device consisting of a stainless steel plate and a knife with a 30 nominal coating height of $100 \mu \mathrm{m}$. The final mix of the reactants is poured on ethylen-tetrafluorethylen (ETFE) foil and the knife is moved smoothly over the foil to form the film. The film is left to cure over night at ambient temperature.

The use of ETFE foil has the advantage of making the very thin 35 films handable and easy to store.

The mechanical characterization of the bimodal networks is performed using a controlled stress rheometer (AR2000) from TA Instruments set to a controlled strain mode with $2 \%$ strain, which is ensured to be within the linear regime of the material based on

40 an initial strain sweep. The linear viscoelastic data are measured with parallel plate $(25 \mathrm{~mm})$ geometry at $25^{\circ} \mathrm{C}$.

A swelling/extraction procedure was applied to determine the sol fraction of some of the networks. The sol fractions were extracted in heptane for 72 hours at room temperature. The heptane was 45 replaced after 48 hours. The networks were then dried overnight. The materials were weighed before $\left(\mathrm{m}_{0}\right)$ and after extraction $\left(\mathrm{m}_{1}\right)$ and the sol fraction was calculated from $\mathrm{W}_{\mathrm{sol}}=\left(\mathrm{m}_{0}-\mathrm{m}_{1}\right) / \mathrm{m}_{1}$.

\section{${ }_{50}$ Results and discussions}

All the determined storage moduli at two frequencies ( 0.001 and $100 \mathrm{~Hz}$, respectively) are shown in table 1 . If the two values are identical it means no viscous dissipation in the frequency interval. Furthermore $\omega_{\text {plat }}$ is given in the table. This is the given frequency 55 at which there is no relaxation (i.e. no viscous dissipation) happening at lower frequencies. This is recorded when there is no change in the storage modulus with decreasing frequency. The elastic moduli are determined from $\mathrm{G}=\mathrm{G}^{\prime}(\omega \rightarrow 0)$. For the unimodal networks the elastic moduli fall in the range between 13 60 to $200 \mathrm{kPa}$ and for the bimodal networks in the range between 10 to $600 \mathrm{kPa}$. The systems with fillers (samples 27, 28) all have significantly higher elastic moduli. All bimodal networks without fillers are handable and can easily be transferred from the release liner which means that the tear strength is acceptable for DEAP

${ }_{65}$ purposes. The prepared unimodal networks tear very easily upon handling and can not be used without addition of reinforcing fillers.

In Figure 7 the elastic behaviour as function of the applied frequency (LVE diagram) is shown for unimodal networks based 70 on PDMS-V35 with different values of r. As expected, the elastic modulus increases with the stoichiometric imbalance and the frequency dependency of $G^{\prime}$ is getting less pronounced due to the reduced fractions of dangling and sol material and hence lower tendency to viscous dissipation. The same trends were observed 75 from the V41 networks. A modulus of as low as $10^{5} \mathrm{~Pa}$ can be obtained (for the systems V35, r=1,03 and V41, r=0,7) but the network contains a large fraction of sol and dangling substructures which gives rise to the dynamic behaviour of $\mathrm{G}^{\prime}(\omega)$. The stoichiometric imbalance ensuring the highest elastic 80 modulus is 1.5 . It is believed to be due to steric hindrance of the crosslinker that the stoichiometry is so far from the ideality where $\mathrm{r}=1$ should ensure the strongest network. It is, however, wellknown that a stoichiometry of $r=1.1-1.3$ usually gives the highest value of the elastic modulus ${ }^{31}$. The unimodal networks with 85 lowest values of $r$ are also extremely fragile and difficult to handle due to low tear strength.

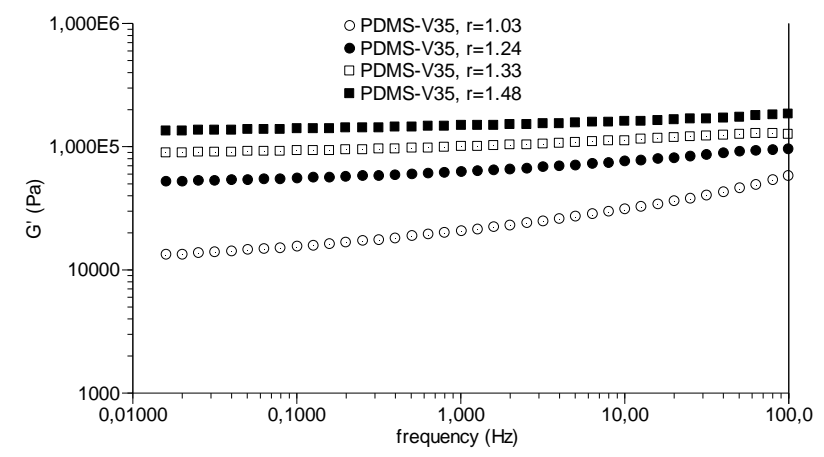

Figure 7. The viscoelastic behaviour of PDMS-V35 based unimodal network

The bimodal networks are synthesized using one of the two methods $(1 \mathrm{~s}-4 \mathrm{p}$ or $2 \mathrm{~s}-4 \mathrm{p})$ and characterized by their rheological properties. Identical long PDMS are used both for unimodal and 95 bimodal networks as the long chain polymer. 
Table 1. Summary of all the synthesized and characterized samples

\begin{tabular}{|c|c|c|c|c|c|c|c|c|c|c|}
\hline $\begin{array}{l}\text { Sample } \\
\text { number }\end{array}$ & Sample code & $\mathbf{M}_{\text {shart }}(\mathrm{g} / \mathrm{mol})$ & $\mathbf{M}_{\text {bag }}(\mathrm{g} / \mathrm{mol})$ & Short:long & $r$ & $\begin{array}{c}\mathrm{G}^{\prime} \text { at } 0.00 \mathrm{lHz} \\
(\mathrm{KPa})\end{array}$ & $\begin{array}{l}\text { G' at } 100 \mathrm{~Hz} \\
(\mathrm{KPa})\end{array}$ & $\frac{\mathrm{G}^{\prime}(100 \mathrm{~Hz})-\mathrm{G}^{\prime}(0.001 \mathrm{~Hz})}{\mathrm{G}^{\prime}(100 \mathrm{~Hz})}$ & $\omega \_$plateau $(\mathrm{Hz})$ & procedure \\
\hline 0 & ElastosilRT 625 & - & - & - & - & 188 & 250 & 0.248 & 10 & - \\
\hline 1 & \multirow{3}{*}{ V05-V35 } & \multirow{3}{*}{800} & \multirow{3}{*}{49500} & \multirow{3}{*}{$80: 20$} & 0.8 & 28 & 29.1 & 0.037 & 25 & \multirow{3}{*}{$2 s-4 p$} \\
\hline 2 & & & & & 1 & 149 & 150 & 0.006 & 50 & \\
\hline 3 & & & & & 1.2 & 329 & 377 & 0.127 & 50 & \\
\hline 4 & \multirow{3}{*}{ V05-V41 } & \multirow{3}{*}{800} & \multirow{3}{*}{62700} & \multirow{3}{*}{$80: 20$} & 0.8 & 26.3 & 32.7 & 0.195 & 0.5 & \multirow{3}{*}{$2 s-4 p$} \\
\hline 5 & & & & & 1 & 184 & 190 & 0.031 & 40 & \\
\hline 6 & & & & & 1.2 & 537 & 567 & 0.052 & 60 & \\
\hline 7 & \multirow{3}{*}{ V22-V41 } & \multirow{3}{*}{9400} & \multirow{3}{*}{62700} & \multirow{3}{*}{$80: 20$} & 0.8 & 7.8 & 13.1 & 0.404 & 0.2 & \multirow{3}{*}{$2 s-4 p$} \\
\hline 8 & & & & & 1 & 22 & 65.6 & 0.664 & 0.015 & \\
\hline 9 & & & & & 1.2 & 135 & 144 & 0.062 & 20 & \\
\hline 10 & V05-V22 & 800 & 9400 & \multirow{3}{*}{$90: 10$} & \multirow{3}{*}{0.8} & 79 & 77.8 & - & 25 & \multirow{3}{*}{$2 s-4 p$} \\
\hline 11 & V05-V41 & 800 & 62700 & & & 36.9 & 48.1 & 0.232 & 0.02 & \\
\hline 12 & V22-V41 & 9400 & 62700 & & & 9.1 & 11.4 & 0.201 & 0.2 & \\
\hline 13 & \multirow{4}{*}{ V05-V35 } & \multirow{4}{*}{800} & \multirow{4}{*}{49500} & $97: 3$ & \multirow{4}{*}{1} & 200 & 211 & 0.052 & 25 & \multirow{4}{*}{$2 s-4 p$} \\
\hline 14 & & & & $90: 10$ & & 141 & 143 & 0.013 & 2 & \\
\hline 2 & & & & $80: 20$ & & 149 & 150 & 0.006 & 50 & \\
\hline 15 & & & & $50: 50$ & & 35.1 & 41.2 & 0.148 & 2 & \\
\hline 16 & V05-V41 & 800 & 62700 & $97: 3$ & 1 & 139 & 143 & 0.027 & 65 & $2 s-4 p$ \\
\hline 17 & & & & $90: 10$ & & 248 & 250 & 0.008 & 80 & \\
\hline 5 & & & & $80: 20$ & & 184 & 190 & 0.031 & 40 & \\
\hline 18 & V05-V41 & 800 & 62700 & $30: 70$ & 1 & 17 & 43.4 & 0.608 & 0.16 & $2 s-4 p$ \\
\hline 19 & & & & $20: 80$ & & 4.2 & 30.2 & 0.860 & 0.04 & \\
\hline 20 & & & & $10: 90$ & & 3.6 & 36.9 & 0.902 & 0.025 & \\
\hline 21 & & & & $97: 3$ & & 173 & 196 & 0.117 & 0.6 & \\
\hline 22 & V22-V41 & 9400 & 62700 & $90: 10$ & 1 & 56 & 62.2 & 0.099 & 0.4 & $2 s-4 p$ \\
\hline 8 & & & & $80: 20$ & & 22 & 65.6 & 0.664 & 0.015 & \\
\hline 23 & & & & $30: 70$ & & 136 & 183 & 0.256 & 0.25 & \\
\hline 24 & V05-V35 & 800 & 49500 & $20: 80$ & 1.2 & 26.6 & 63.3 & 0.579 & 0.13 & $2 s-4 p$ \\
\hline 25 & & & & $10: 90$ & & 19.1 & 30.1 & 0.365 & 0.015 & \\
\hline 25 & V05-V35 & 800 & 49500 & 10:90 & 1.2 & 19.1 & 30.1 & 0.365 & 0.015 & $2 s-4 p$ \\
\hline 26 & & & & $10: 90$ & & 84.7 & 104 & 0.185 & 0.2 & $1 s-4 p$ \\
\hline 27 & V05-V35 & 800 & 49500 & $20: 80$ & 1.2 & 52.1 & 66 & 0.210 & 0.04 & $+10 \% \mathrm{SiO}_{2}$ \\
\hline 28 & & & & & 0.8 & 91.1 & 97 & 0.060 & 0.2 & \\
\hline 29 & & & & & 1.03 & 13.1 & 57.7 & 0.772 & - & \\
\hline 30 & V35 & - & 49500 & - & 1.24 & 51.6 & 95.2 & 0.457 & 0.4 & - \\
\hline 31 & & & & & 1.33 & 89.3 & 128 & 0.302 & 1 & \\
\hline 32 & & & & & 1.48 & 135 & 179 & 0.245 & 1 & \\
\hline 33 & & & & & 0.7 & 22.5 & 78.1 & 0.711 & - & \\
\hline 34 & V41 & - & 62700 & - & 1.17 & 30.8 & 83.3 & 0.630 & - & - \\
\hline 35 & & & & & 1.22 & 75.4 & 141 & 0.465 & - & \\
\hline
\end{tabular}


PDMS-V05 and PDMS-V22 are used as short chain polymer. The stoichiometric imbalance and the short:long ratio are varied. The stoichiometry of the pre-mixes is kept constant but as close as ${ }_{5}$ possible to the critical value of $r$. The value of $r$ in the premixes could also be varied but in this study we focus on the two extremes, namely the one-step four-pots and the two-steps fourpots have the $\mathrm{r}=0$ and $\mathrm{r} \rightarrow \mathrm{r}_{\mathrm{c}}$ in the premixes, respectively.

In Figure 8 the elastic behaviour of two chemically identical 10 networks prepared by the two different reaction schemes are shown. Elastic moduli of $88 \mathrm{KPa}(1 \mathrm{~s}-4 \mathrm{p})$ and $19 \mathrm{KPa}(2 \mathrm{~s}-4 \mathrm{p})$ are obtained with no significant viscous dissipation in the investigated frequency regime. It is obvious that the more complicated reaction procedure $(2 s-4 p)$ is worthwhile as the 15 modulus is decreased by a factor of 4,5 compared to the traditional mixing procedure $(1 \mathrm{~s}-4 \mathrm{p})$.

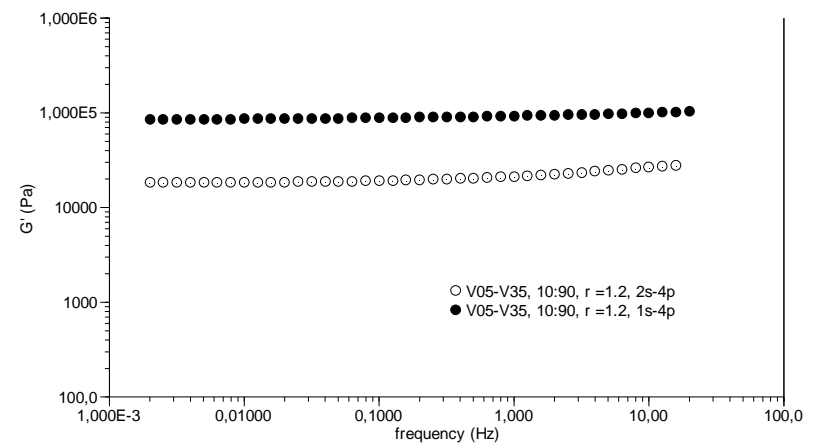

Figure 8 . The viscoelastic behaviour of chemically identical networks synthesized using the $1 \mathrm{~s}-4 \mathrm{p}$ or $2 \mathrm{~s}-4 \mathrm{p}$ procedures, respectively.

Considering the requirements of soft materials for actuators, the difference between the values of $G$ ' for each of the two 25 procedures $(1 \mathrm{~s}-4 \mathrm{p}$ and $2 \mathrm{~s}-4 \mathrm{p})$, all following bimodal networks were synthesized using $2 \mathrm{~s}-4 \mathrm{p}$ procedure.

Furthermore in the rheological characterization of the bimodal networks, the stoichiometric imbalance is varied $(0.8,1,1.2)$ and the mass ratio short:long is kept constant $(80: 20)$, so the influence 30 of PDMS chain length may be analysed (samples 1-3). The behaviour is shown in Figure 9.

For all types of bimodal networks (samples 1 to 28) the values of the elastic moduli increase with increasing $r$ as expected since the sol and dangling arm fractions will be reduced with increasing $r$. 35 It is also evident that the tendency to viscous dissipation within the investigated frequency domain is limited even for networks with low elastic moduli. So, the introduction of the heterogeneity clearly leads to a non-contrary relation between low elastic modulus and little viscous dissipation.

${ }_{40}$ The low elastic moduli of the bimodal networks are very promising, especially the V22-V41 networks, since as discussed previously the bimodal networks will resemble the unimodal short-chain network the most in the low-strain limit (LVE region) and hence it can be expected that the material will be even softer 45 in the high-strain limit ${ }^{3}$.

The rheological behaviour of bimodal networks synthesized with different molecular weights of the polymer was also investigated (samples 10 - 12).

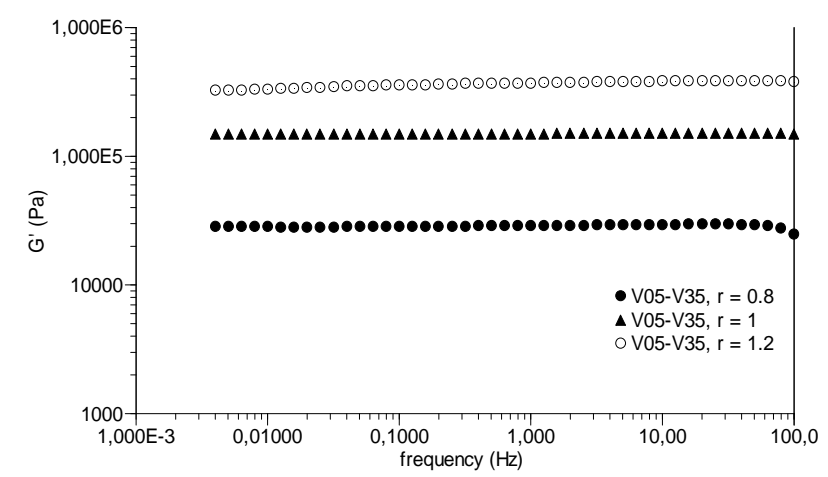

Figure 9. The viscoelastic behaviour of V05-V35 based network with a short:long mass ratio of 80:20 and different $r$ values

The stoichiometric imbalance $(\mathrm{r}=0.8)$ and the short:long ratio $55(90: 10)$ are kept constant. PDMS-V05 is mainly used as short chain, while PDMS-V22 is used both as long and short chain (V05-V22 and V22-V41).

Despite the difference in the molecular weight of PDMS-V35 $(49500 \mathrm{~g} / \mathrm{mol})$ and PDMS-V41 $(62700 \mathrm{~g} / \mathrm{mol})$, no significant differences between the elastic moduli are observed. This can be explained from the molecular weights of the two polymers, which both are significantly above the molecular entanglement weight of PDMS $(12 \mathrm{~kg} / \mathrm{mol})^{32}$ and hence the elastic behaviour of both systems will be dominated by entanglements rather than crosslinks.

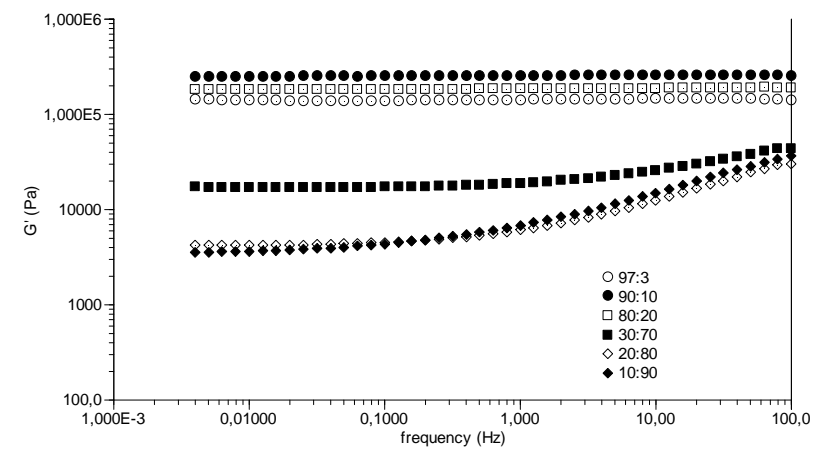

Figure 10. The viscoelastic behaviour of V05-V41 based network $60 \quad(r=1)$. The short:long chain ratio is varied from $97: 3$ to $10: 90$

In figure 10, V05-V41 based bimodal networks are compared with the mass ratio short:long chains varying at stoichiometric conditions $(\mathrm{r}=1)$. This way the crosslink density is varied while 65 the relative amounts of network fractions are identical. The elastic modulus decreases with increased long chain fraction but the significant effect, however, seems rather insignificant at high mass ratios. Viscous dissipation is observed when the ratio is taken bellow 80:20

70 Using PDMS-V22 as short chain and PDMS-V41 as long chain in synthesizing stoichiometric networks (samples 8, 21, 22), the elastic modulus decreases even when the short chain ratio is high $(97: 3,90: 10$ or $80: 20)$. It can be explained by the cluster chains being longer and giving cause to relaxation.

75 Nonstoichiometric bimodal networks (V05-V35, r=1.2) with different mass ratio between the short and long chains have been 
rheologically characterized (samples $23-25$ ). Again very low elastic moduli can be obtained.

The tear strength of the unfilled bimodal networks is low compared to tear strength of commercial filled elastomers such as ${ }_{5}$ ElastosilRT-625 from Wacker Chemie but on the other hand they have a much higher tear strength than unimodal networks with identical elastic modulus arising from a large deficiency in crosslinker. The handability of the bimodal networks is also favored to that of the unimodal networks with identical elastic 10 moduli due to the nonsticky nature of the bimodal networks where in contrast the unimodal networks will be extremely sticky. The bimodal networks have sufficiently high tear strength to be used in microfabrication as they can easily be removed from the spin coater even for very thin films. However, unimodal networks 15 without fillers are more or less impossible to remove without tearing the films. If a higher dielectric strength of the material is needed then it is possible to add small amounts of $\mathrm{BaTiO}_{3}{ }^{33}$. It has been shown that it is possible to increase the dielectric permittivity of the material by $33 \%$ with the addition of $3 \mathrm{w} / \mathrm{w} \%$ 20 of $\mathrm{BaTiO}_{3}$ without altering the rheological properties (within experimental uncertainty) ${ }^{34}$.

The amounts of sol were determined to be less than $10 \%$ for all the investigated bimodal networks V05-V35 and V05-V41, which agrees very well with the sol fractions from unimodal networks.

\section{${ }_{25}$ Conclusions}

It is shown that it is possible by a controlled reaction procedure to synthesize silicone networks with a very low elastic modulus (20$30 \mathrm{kPa}$ ) without introducing any significant viscous dissipation. This has been attempted previously by increasing the molecular 30 weight of the polymers but the effect is limited due to entanglements, and if the stoichiometry is used as the optimization parameter, networks with low elastic modulus can be obtained but they have a significant viscous loss. The positive effect arises because the reaction procedure allows the network to 35 form stepwise. First clusters of two polymers with different molecular weights are allowed to form by reaction with crosslinker in deficiency to ensure that no gelation occurs. Then the two different clusters are assembled by reaction with crosslinker. This way heterogeneity in the network topology is 40 introduced. The domains with the clusters of small polymers act as a reinforcing structure of the soft network from the long polymers.

\section{Aknowledgements}

The authors would like to acknowledge the financial support 45 from Danish Agency for Science, Technology and Innovation and the Danish National Advanced Technology Foundation.

\section{Notes and references}

${ }^{a}$ Søltofts Plads, Building 229, 2800 Kgs. Lyngby, Denmark, Fax: 004545256198, Tel: 004545256885, agb@kt.dtu.dk

${ }_{50}{ }^{b^{*}}$ Søltofts Plads, Building 229, 2800 Kgs. Lyngby, Denmark, Fax: 004545882258,Tel: 004545252825, al@kt.dtu.dk

1. A. L. Larsen, K. Hansen, P. Sommer-Larsen, O. Hassager, A. Bach, S. Ndoni and M. Jorgensen, Macromolecules, 2003, 36 , 10063-10070.
2.

604.

5.
A. L. Larsen, P. Sommer-Larsen and O. Hassager, in Smart Structures and Materials 2004 Conference, San Diego, CA, 2004, pp. 108-117.

J. E. Mark, Macromolecular Symposia, 2003, 191, 121-130.

J. E. Mark, Progress in Polymer Science, 2003, 28, 12051221.

Y. Bar-Cohen, Electroactive Polymer (EAP) Actuators as Artificial Muscles: Reality, Potential and Challenges, Bellingham, WA, 2004.

R. Pelrine, J. Eckerle and S. Chiba, in Third International Symposium on MicroMachine and Human Science, Nagoya, Japan, 1992.

M. Zhenyl, J. I. Scheinbeim, J. W. Lee and B. A. Newman, Journal of Polymer Science, Part B: Polymer Physics, 1994, 32, 2721-2731.

Q. Zhang, V. Bharti and X. Zhao, Science 1998, 280, 21012104.

R. Pelrine, R. Kornbluh, Q. Pei and J. Joseph, Science 2000, 287, 836-839.

R. Pelrine, R. Kornbluh and G. Kofod, Advanced Materials, 2000, 12, 1223-1225.

K. Meijer, M. Rosenthal and R. Full, in Smart Structures and Materials Conference, Newport Beach CA 2001.

G. Kofod, Journal of Physics D-Applied Physics, 2008, 41.

Y. Bar-Cohen, S. Leary, M. Shahinpoor, J. Harrison and J. Smith, Proc. SPIE, 1999, 3669, 57-63.

4. Kornbluh, H. Prahlad and R. Pelrine, in Smart Structures and Materials 2004 Conference, San Diego, CA, 2004, pp. 372-386.

R. Shankar, T. Ghosh and R. Spontak, Soft. Matter., 2007, 3, 1116-1129.

R. E. Pelrine, R. D. Kornbluh and J. P. Joseph, Sensors and Actuators a-Physical, 1998, 64, 77-85.

J. Plante and S. Dubowsky, Smart Materials Structures 2007, 16, 227-236.

J. Plante and S. Dubowsky, International Journal of Solids and Structures, 2006, 43, 7727-7751.

J. D. Madden, N. A. Vandesteeg, P. A. Anquetil, P. G. A. Madden, A. Takshi, R. Z. Pytel, S. R. Lafontaine, P. A. Wieringa and I. W. Hunter, IEEE J. Oceanic Eng., 2004, 29, 706-728.

B. Balakrisnan and E. Smela, in Conference on Electroactive Polymer Actuators and Devices (EAPAD) 2010, San Diego, CA, 2010.

M. K. Jensen, H. K. Rasmussen, A. Bach, A. L. Skov, O. Hassager and H. Koldbech, Rheologica Acta, 2010, 49, 1-13. A. Bejenariu, H. K. Rasmussen, A. L. Skov and O. Hassager, Rheologica Acta, 2010, 49, 807-814.

M. K. Jensen, H. K. Rasmussen, A. L. Skov and O. Hassager, Rheologica Acta, 2011, 50, 729-740.

4. K. Jensen, A. Bach, O. Hassager and A. L. Skov, International Journal of Adhesion and Adhesives, 2009, 29, 687-693.

G. Ouyang, K. Wang, M. N. Akram, L. Henriksen and X. Y. Chen, Applied Physics a-Materials Science \& Processing, 2011, 103, 381-388. 
26. S. M. Grundahl Frankær, M. Krog Jensen, A. G. Bejenariu and A. Ladegaard Skov, Rheologica acta, submitted.

27. M. Benslimane, M. J. Tryson, J. Oubak and H.-E. Kiil, in Conference on Electroactive Polymer Actuators and Devices (EAPAD) 2011, San Diego, CA, 2011.

28. A. Larsen, K. Hansen, P. Sommer-Larsen, O. Hassager, A. Bach, S. Ndoni and M. Jorgensen, Macromolecules, 2003, 36, 10063-10070.

29. P. J. Flory, Principles of Polymer Chemistry, 1953.

10 30. W. H. Stockmayer, Journal of Chemical Physics, 1944, 12 , 125-131.

31. A. L. Larsen, P. Sommer-Larsen and O. Hassager, EPolymers, 2004

32. L. J. Fetters, D. J. Lohse, D. Richter, T. A. Witten and A. 15 Zirkel, Macromolecules, 1994, 27, 4639-4647.

33. E. A. Cherney, 2005 Annual Report Conference on Electrical Insulation and Dielectric Phenomena, 2005, 1-9.

34. A. D. Egede, A. G. Bejenariu, J. Bøgelund, M. Benslimane and A. L. Skov, Proceedings of SPIE 2012, to be submitted, 2012. 